\title{
TAGUNG
}

\section{Transatlantische Lastenteilung: Wer trägt die Kosten für die Bewahrung globaler Sicherheit?}

\author{
Katharina Plück*
}

Die globale Sicherheitsordnung ist ein Anliegen, das die westlichen Alliierten teilen trotz der außenpolitischen Meinungsverschiedenheiten zwischen den transatlantischen Partnern, die nach Ende des Kalten Krieges und spätestens nach dem 11. September 2001 sowie dem amerikanischen Einmarsch in den Irak im März 2003 deutlich zugenommen haben. Vor dem Hintergrund eines drohenden nuklearen Waffenprogramms im Iran, kontinuierlicher Terror-Aktivitäten im Irak und erneut aufflammender Gewalt in Afghanistan erscheint das Zusammenwirken des Westens in Sachen weltweiter Sicherheit wichtiger denn je. Natürlich ist es nicht damit getan, sich auf ein so umfassendes und ehrgeiziges Ziel wie die Bewahrung globaler Sicherheit zu einigen. Vielmehr müssen sich die westlichen Partner darüber einig werden, wie die Verantwortung und die damit verbundenen Lasten für diese gemeinsame außenpolitische Aufgabe verteilt werden können.

Weltweite, langfristig aufrechterhaltene militärische Präsenz beruht auf wesentlichen ökonomischen Voraussetzungen. Zum einen müssen Länder, die eine globale Führungsrolle für sich beanspruchen (oder von Partnern mit dieser beauftragt werden), über eine gesunde, wachsende Volkswirtschaft verfügen. Zum anderen erfordern moderne, weltweit einsatzfähige Streitkräfte erhebliche wirtschaftliche Ressourcen - nicht zuletzt für Forschung und Entwicklung, die die Basis einer technologisch führenden Rüstungsindustrie und somit der angemessenen Ausstattung des Militärs darstellt. Das Aufbringen dieser Mittel wie-

\section{Sharing the Growing Economic Burden of World Order: How to strengthen the economic base of foreign and security policy in the} Atlantic Alliance?

Internationale Konferenz des Arbeitskreises Europäische Integration e.V., der Stiftung Wissenschaft und Politik (SWP) und des Institute for International Economics (Washington, DC)

Berlin, 11./12. Mai 2006

Welcome and Introduction

Dr. Stefan MAIR, Mitglied der Institutsleitung, SWP Dr. Jens VAN SCHERPENBERG, Leitung, Forschungsgruppe Amerika, SWP

The challenge: The U.S. as the provider of last resort of global security - which place for Europe?

The political reasons for global economic imbalances: Why Europe finances American military activities abroad and economic consumption at home Dr. Henry NAU, George Washington University, Washington, DC

Comment: Karl-Theodor ZU GUTTENBERG, $\mathrm{MdB}$

Overcoming rising budgetary risks and their foreign and security policy implications

Guns and butter - How long can it work? The political economy of U.S. security policy

Dr. Peter DOMBROWSKI, Naval War College, Newport, RI

How to win votes with sound budgetary and tax policies?

Dr. Kevin HASSETT, American Enterprise Institute, Washington, DC

Comment: Dr. Bernhard SPEYER, Deutsche Bank Research

* Katharina Plück, M.A., wiss. Mitarbeiterin am Institute for International Economics, Washington, DC. Die Autorin dankt Dr. Jens van Scherpenberg für die Unterstützung bei der Erstellung des Tagungsberichts. 
derum bedarf in einer Demokratie der Zustimmung oder Duldung der Bürger. Sie müssen das außenpolitische Engagement ihres Landes unterstützen. Wenn die öffentliche Meinung dagegen vor allem auf innenpolitische Themen fixiert ist, werden Politiker es schwer haben, ausreichend Gelder für Außen- und Sicherheitspolitik zu mobilisieren.

Der klassische Konflikt, dem sich politische Führungskräfte gegenüber sehen, nämlich zwischen ,Kanonen und Butter', der Stärkung der äußeren Sicherung des Landes und der inländischen Leistungen für die Bürger entscheiden zu müssen, wurde durch die Liberalisierung der internationalen Finanzflüsse in den siebziger und achtziger Jahren gemindert. Dank der Öffnung nationaler Kapitalmärkte können Länder trotz eigener Ressourcenknappheit leichter Mittel auf internationalen Finanzmärkten leihen und damit sowohl inländischen Anforderungen wie auch außenpolitischen Ambitionen entsprechen. Allerdings ließ sich der Zielkonflikt nie gänzlich aufheben. Eine wachsende Außenverschuldung beinhaltet, dass nachfolgende Generationen Schulden tilgen und auf Teile des erwirtschafteten Inlandsproduktes verzichten müssen. Das bedeutet auch, dass weniger Mittel für zukünftige inländische und außenpolitische Ziele vorhanden sind; mehr noch: Die entstehenden globalen wirtschaftlichen Ungleichgewichte bergen ihre eigenen Gefahren für die Stabilität der Weltwirtschaft und somit auch für die Bewahrung globaler Sicherheit.

Mit einem Leistungsbilanzdefizit von mittlerweile fast sieben Prozent des Bruttoinlandproduktes haben die Vereinigten Staaten in den letzten Jahren einen Immobilienboom und bedeutende militärische Einsätze finanziert, und dies bei einer negativen Sparquote der amerikanischen Privathaushalte in 2005. Die meisten europäischen Partner und Japan verzeichnen dagegen Bilanzüberschüsse, langsames Wirtschaftswachstum und zugleich konstante Verteidigungsausgaben. Diese Länder sind dadurch nicht nur auf Amerikas globale Schutzfunktion angewiesen, ihr - wenngleich zaghaftes - wirtschaftliches Wachstum hängt
When the U.S. current account deficit cannot be sustained anymore

Who is going to foot the bill? Economic consequences of a disorderly dollar adjustment for the U.S., Europe and Asia

Dr. Sebastian DULLIEN, Financial Times Deutschland

Adjustment challenges for the United States

Dr. Adam POSEN, Institute for International Economics, Washington, DC

Comment: Prof. Dr. Carl-Ludwig HOLTFRERICH, Freie Universität, Berlin

How resilient is the U.S. Economy?

The United States as net debtor: how much longer the "Exorbitant Privilege“?

Dr. Catherine MANN, Institute for International Economics, Washington, DC

Katharina PLÜCK, Institute for International Economics, Washington, DC

The attractiveness of U.S. financial markets: the example of the housing sector

Martin MÜHLEISEN, International Monetary Fund, Washington, DC

Can technology power American leadership: meeting the challenges of the 21st century

Dr. Kent HUGHES, Wilson Center for International Scholars, Washington, DC

Comment: Stormy MILDNER, M.Sc., Freie Universität, Berlin

Paying up to share responsibilities: Europe's role and realities - a mixed picture with a silver lining?

Burden-sharing and strategic policy options for Europe

Dr. Stefan COLLIGNON, Harvard University, Cambridge, MA

Foreign Policy spending in the EU - Recent trends and explanations

Dr. des. Daniela SCHWARZER, SWP

Comment: Dr. Tilman BRÜCK, Deutsches Institut für Wirtschaft, Berlin

Conclusion: The task - Forging a new transatlantic burden sharing consensus

How much scope for shared responsibilities in the Atlantic Alliance?

Dr. Steven SZABO, Johns Hopkins University School of Advanced International Studies, Washington, DC

Redressing the world's imbalance? European public discourses and the prospects for transatlantic burden-sharing

Prof. Dr. Reinhard WOLF, Ernst-Moritz Arndt Universität, Greifswald 
in entscheidendem Maße von der Fähigkeit der amerikanischen Wirtschaft $a b$, eine steigende Menge ausländischer Einfuhren zu absorbieren. Ist eine solche Situation auf Dauer tragbar?

Diese Frage beschäftigte die Teilnehmer der eineinhalb-tägigen Konferenz "Sharing the growing economic burden of world order" in Berlin. Werden amerikanische Wähler das intensive außenpolitische Engagement ihres Landes weiterhin unterstützen? Kann die amerikanische Wirtschaft in der Zukunft ein solches Engagement tragen? Können und wollen Europa, Japan und andere Alliierte die Verantwortung für die internationale Sicherheit weiterhin im bisherigen Umfang den Vereinigten Staaten überlassen? Wenn nicht, was muss geschehen, damit die Kosten dieser globalen Sicherheitsfunktion gleichmäßiger verteilt werden?

Die Verknüpfung von wirtschaftlichen Fragen und außen- sowie sicherheitspolitischen Problemen wird oft diskutiert, wenn Themen wie Handelssanktionen oder internationale Energiepolitik zur Debatte stehen. Der Versuch jedoch, die Verbindungen zwischen inländischer Wirtschaftspolitik, internationalen makroökonomischen Entwicklungen und globaler Sicherheits- und Verteidigungspolitik nachzuzeichnen, sei nach Angaben des Konferenzleiters Jens van Scherpenberg relativ neu. Obwohl diese Verflechtungen zum politischen Alltag gehörten seien sie von Wissenschaftlern noch wenig erforscht. Die Konferenz könne deshalb auch keine konkreten Antworten versprechen, aber Denkanstöße liefern und zu weiterer Diskussion anregen.

\section{Die Vereinigten Staaten, Europa und der Sta- tus Quo}

In seinem einleitenden Beitrag stellte Henry Nau heraus, dass die gegenwärtige wirtschaftliche und außenpolitische Kräftekonstellation in der Welt aus rein geopolitischer Sicht auf unbestimmte Zeit fortbestehen werde, da sie im Interesse aller Beteiligten sei. Er zeigte, dass das amerikanische Leistungsbilanzdefizit in den letzten 15 Jahren trotz unterschiedli- cher Wirtschaftspolitik verschiedener Administrationen und trotz erheblicher Schwankungen im Wechselkurs des US Dollars stetig gewachsen ist. Völlig unabhängig von Zielen der amerikanischen Wirtschaftspolitik seien die Partner der USA offenbar bereit, ihre Ersparnisse in die amerikanische Wirtschaft zu investieren. Grundlage dafür sei jedoch, dass die Vereinigten Staaten mit weltweit militärischer Präsenz globale Sicherheit garantieren könnten und der amerikanische Markt der Motor der Weltwirtschaft bleibe. Die Konstellation liege also auch im innenpolitischen Interesse der Partnerländer. Nau bemerkte, dass die Abhängigkeit der USA von ausländischen Kapitalzuflüssen die amerikanische Außenpolitik bisher nicht beeinflusst habe. Vielmehr sei die derzeitige amerikanische Führung unilateraler in ihrer Ausrichtung als viele ihrer Vorgänger. Die Leistungsüberschuss-Länder hätten implizit die amerikanische Wirtschaftsund Außenpolitik bestätigt.

Solch eine Interdependenz lasse sich wahrscheinlich nur durch größere Schocks auflösen: Zum Beispiel eine Finanz- und Bankenkrise in China, ausgelöst durch ineffiziente Überinvestionen, Spannungen zwischen den transatlantischen Partnern und der Volksrepublik in Sachen Asien oder ein Konflikt mit Russland in Bezug auf die Entwicklungen im Nahen Osten. Ebenso hätte ein Zusammenbruch der Beziehungen zwischen Europa und den USA gravierende Konsequenzen.

Allerdings kann Nau sich zunächst bestenfalls schrittweise Veränderungen vorstellen, die zu einer ausgeglicheren Lastenverteilung in dem Bemühen um globale Sicherheit führen könnten, zumal es momentan keine Alternativen für den Status Quo gebe: Europa habe sich schon immer als Zivilmacht gesehen und scheine nicht bereit, in den nächsten Jahren eine größere militärische Rolle in der Welt zu übernehmen. Ein Rückzug der USA könne zu einem gefährlichen Machtvakuum führen und sei sicherlich nicht im Interesse der Europäer. Außerdem gebe es für ausländische Investoren keine attraktiveren Zielorte als die flexible und 
dynamische amerikanische Volkswirtschaft. Seit 2001 beträgt die jährliche Wachstumsrate der Produktivität in den Vereinigten Staaten 3,6 Prozent; während das Produktivitätswachstum in Deutschland und Japan bei einer jährlichen Rate von 1,2 beziehungsweise 1,1 Prozent liegt. Amerikanische Finanzmärkte arbeiten effizienter; die USA verfügen über das beste Justizsystem in der Welt und gewährleisten den stärksten Schutz gewerblicher Urheberrechte, sagte Nau. Bevor Europa und Japan ihre Dienstleistungssektoren deregulierten und vor allem ihre Finanzmärkte reformierten, erwarte er keine großen Veränderungen. Zwar sei das derzeitige Leistungsbilanzdefizit der USA beispiellos, aber ein Niveau von etwa drei Prozent des Bruttoinlandproduktes sei auf lange Sicht durchaus vorstellbar.

Karl-Theodor zu Guttenberg stimmte mit Nau überein, dass Europas Ausblick zu sehr auf seine eigene Grenzen beschränkt sei und $\mathrm{Eu}-$ ropäer dem transatlantischen Verhältnis $\mathrm{zu}$ sehr verhaftet seien. Amerika habe sich stattdessen viel früher dynamischeren Märkten wie den Volkswirtschaften Indiens und Chinas zugewandt. Europas Rolle in der Welt habe durch die Verfassungskrise sehr gelitten, und es helfe nicht, dass das Paradox von gleichzeitiger Erweiterung und Vertiefung immer noch nicht gelöst sei. Eines der wichtigsten Hindernisse sei eben Europas Unvermögen, ein eigenes „europäisches Interesse“ zu definieren. Der Vorschlag des Verfassungsentwurfes, den Posten eines gemeinsamen europäischen Außenministers zu schaffen, müsse unbedingt verwirklicht werden. Auch müsse die Europäische Verteidigungsagentur effektiver gemacht werden, um weitere ineffiziente Doppelungen in nationalen Verteidigungsprogrammen zu verhindern.

Trotzdem, so zu Guttenberg, habe Europa in den letzten Jahren durchaus konkrete Beiträge zur Bewahrung der globalen Sicherheit geleistet. Als Beispiele nannte er den bevorstehenden EU-Einsatz im Kongo, das Engagement in Darfur und die bedeutende deutsche militärische Präsenz in Afghanistan. Zu Gut- tenberg zeigte sich auch hoffnungsvoll, was die zukünftige Entwicklung der Nato angehe. Die Bundesregierung habe mehrfach bekräftigt, dass die transatlantische Organisation in europäischen Verteidigungsplänen Vorrang haben solle. Er glaube auch, dass das Bemühen um einen transatlantischen Lastenausgleich am besten in der Nato aufgehoben sei. Neben ,Berlin-Plus' könne man sich zum Beispiel ein umgekehrtes ,Berlin-Plus ' vorstellen, das es der Nato ermögliche, häufiger auf Europas zivile Hilfsmittel zurückzugreifen.

\section{Kanonen oder Butter: Haushaltskrise in den USA}

Dass Europa so häufig die zivile und den USA die militärische Rolle zugewiesen wird liegt daran, dass die militärischen Mittel der Vereinigten Staaten die der Europäer weit übersteigen. Peter Dombrowski zeigte in seinem Beitrag, dass amerikanische Verteidigungsausgaben derzeit ebenso viel ausmachen wie die Summe der Verteidigungsausgaben der dreißig Staaten mit dem nächst größten Militäraufwand. Im Verhältnis zum Bruttoinlandsprodukt seien die gegenwärtigen amerikanischen Ausgaben zwar kleiner als während des Kalten Krieges, allerdings nähmen Verteidigungsmittel einen immer größer werdenden Teil der jährlich festzusetzenden (diskretionären) Staatsausgaben ein, deren Anteil am Staatshaushalt selbst seit einigen Jahren zugunsten der gesetzlichen Leistungsausgaben (mandatory spending) abnimmt.

$\mathrm{Ob}$ die militärische Vormachtstellung der USA in den nächsten Jahren erhalten bleibe hänge von vielen Faktoren ab, so Dombrowski. Zwar könnte sich ein ebenbürtiger Konkurrent entwickeln, der die Position der USA in Frage stellen würde, allerdings halte er diese Möglichkeit für unwahrscheinlich. Andere Länder könnten sich in einer anti-hegemonischen Allianz zusammenschließen, um ein Gegengewicht zu den Vereinigten Staaten zu bilden. Die Wirtschaft der USA könnte schwächer werden. Und schließlich könnten strategische Fehlberechnungen die 
amerikanische Vorrangestellung gefährden. Laut Dombrowski war eine solche Fehlberechnung der Krieg im Irak. Offizielle Schätzungen des Verteidigungsministeriums hatten die Kosten der Invasion und Besetzung auf etwa 330 Milliarden Dollar beziffert. Mittlerweile sagten Wissenschaftler aber, dass die Kosten eher bei einer oder zwei Billionen Dollar lägen. Vor diesem Hintegrund sei es fraglich, ob Amerikaner wachsende Militärausgaben und ein weiterhin hohes außenpolitisches Engagement ihres Landes unterstützen würden. Dombrowski nannte die Ergebnisse einer Umfrage, nach der die Mehrheit der Befragten höhere Staatsausgaben für Gesundheit und Bildung höheren Militärausgaben vorzögen. Die Lasten zur Bewahrung globaler Sicherheit würden auf Dauer nicht von amerikanischen Steuerzahlern getragen werden.

Was den Spielraum für die amerikanischen Militärausgaben und andere diskretionäre Staatsausgaben auf die Dauer einschränken wird, sind die stetig zunehmenden gesetzlichen Leistungsverpflichtungen im amerikanischen Staatshaushalt, vor allem für Sozialrenten und Gesundheitswesen. Laut Kevin Hassett liegt der Gegenwartswert dieser Verbindlichkeiten bei mittlerweile zehn Billionen US Dollar. Sie entsprechen also fast dem gesamten jetzigen Bruttoinlandsprodukt der USA. Die amerikanische Regierung habe ihren älteren Wählern fast alle zukünftigen Ressourcen des Landes versprochen, sagte Hassett. Die amerikanische Steuerordnung, das Haushaltsdefizit und versprochene Rentenanwartschaften sowie Verbindlichkeiten in der gesetzlichen Krankenversicherung seien nicht zu verteidigen.

Schuld an dieser unhaltbaren Situation sei, so Hassett, der amerikanische „Mittel-Wähler“, der sich in den letzten Legislaturperioden beachtliche Steuersubventionen und Transfers von zukünftigen Generationen „herbeigewählt" habe. Sowohl Demokraten als auch Republikaner hätten ihre Wahlkampagnen auf diese „Mittel-Wähler“ eingestellt. Und kein Politiker, der auf Wiederwahl hoffe, könne es sich leisten, gegebene Wahlversprechen rückgängig zu machen. Hassett spekulierte, dass die einzige Möglichkeit, verantwortliche Wirtschafts- und Haushaltspolitik zurück auf die Tagesordnung des Kongresses zu bringen, ein internationales Abkommen ${ }^{1}$ über die $\mathrm{Ge}-$ staltung einer solchen verantwortlichen Politik sei. International festgelegte Haushaltsziele könnten es heimischen Politikern ermöglichen, ihre Wähler von nötigen Einschnitten zu überzeugen. Hassett bemerkte, dass ein „faschistisch-kapitalistischer" Staat wie China nicht am „Mittel-Wähler“-Problem leide und deshalb wirtschaftlich erhebliche Vorteile genieße.

Bernhard Speyer unterstrich in seinem Kommentar zu Dombrowskis und Hassetts Vorträgen, dass das gegenwärtige Haushaltsdefizit der USA vor dem Hintergrund der enormen Dynamik einer elf Billionen Dollar großen Volkswirtschaft gesehen werden müsse. Gegenwärtige Steuersätze seien so niedrig, dass sie ohne weiteres angehoben werden könnten, ohne dabei das wirtschaftliche Wachstum zu gefährden. Und die diskretionären Staatsausgaben seien in dieser Legislaturperiode bereits stark gekürzt worden. Auf lange Sicht jedoch könnten diese Kürzungen nicht weiter fortgeführt werden, da diskretionäre Staatsausgaben Aufwendungen für Infrastruktur, Grundlagenforschung, und Bildung enthielten und für die Ausschöpfung des Wachstumspotenzials der Wirtschaft sehr wichtig seien. Speyer wies auch darauf hin, dass es langfristig von Bedeutung sei, wer genau die Inhaber amerikanischer Schulden seien. Wenn die öffentlichen Schulden hauptsächlich von ausländischen Investoren und vor allem ausländischen Zentralbanken finanziert würden, schaffe das eine instabile Interdependenz zwischen den USA und seinen ausländischen Partnern.

1 Hassetts Titel für ein solches Abkommen wäre, Entitlement-Kyoto'. 
Ähnlich wie Speyer hielten andere Konferenzteilnehmer die amerikanische Haushaltspolitik für nicht so aussichtslos wie Hassett sie geschildert hatte. Vor allem bei den Renten könne man Kürzungen vornehmen, die von den Wählern wenig wahrgenommen würden. Auch sei der Wähler es gewohnt, dass Politiker Versprechungen machten, die sie anschließend nicht hielten. Allerdings erhofften sich einige Teilnehmer, dass es charismatischen Führungspersonen gelingen könnte, die Probleme klar und deutlich darzustellen und die Bürger so von der Wichtigkeit von Haushaltsreformen $\mathrm{zu}$ überzeugen. Allerdings müsse dies geschehen bevor der Hauptteil der Wähler über siebzig und verrentet sei.

Die Korrektur von Ungleichgewichten: Szenarien für die USA und den Rest der Welt

Falls sich die gegenwärtigen wirtschaftlichen Ungleichgewichte in der Welt als unhaltbar herausstellen, lässt sich schwer voraussagen, wie eine Rückführung des amerikanischen Leistungsbilanzdefizits aussehen könnte und welche Konsequenzen sie für das weltweite Wachstum hätte. Sebastian Dullien ging in seinem Vortrag davon aus, dass ein amerikanisches Leistungsbilanzdefizit von sieben Prozent des Bruttoinlandproduktes auf die Dauer nicht tragbar sei. Ein Leistungsbilanzdefizit dieser Höhe setze eine völlig unrealistische, eventuelle Außenverschuldung voraus. Eher könne er sich ein Leistungsbilanzdefizit von zwei oder 2,4 Prozent des amerikanischen Bruttoinlandproduktes vorstellen, weil ein Niveau dieser Größenordnung letztendlich eine realistische Stabilisierung der Außenverschuldung ermögliche.

Eine Veränderung im Leistungsbilanzdefizit in einer Größenordnung von über drei Prozent des amerikanischen Bruttoinlandproduktes könne zum Beispiel durch eine Verlangsamung des Konjunkturzyklus in den Vereinigten Staaten ausgelöst werden. Eine andere Entwicklung, die zur Abwertung des Dollarwechselkurses und somit zur Berichtigung des Handelsdefizits führen kann, sei natürlich eine Veränderung in der chinesischen Wechselkurspolitik. Diese würde es auch anderen asiatischen Staaten erlauben, die Unterbewertung ihrer Währungen zu korrigieren. Laut Dullien könne die amerikanische Wirtschaft eine so wesentliche Veränderung des Dollarkurses ohne weiteres verkraften. Allerdings würden die Volkswirtschaften der amerikanischen Handelspartner mit einem Abfall der amerikanischen Nachfrage für ihre Exporte zu kämpfen haben. Ein Rückgang der amerikanischen Nachfrage für Einfuhren in einer GröBenordnung von 440 bis zu 500 Milliarden Dollar entspreche etwa sechs Prozent des gesamteuropäischen Bruttoinlandproduktes.

Welche Konsequenzen eine solche weltweite Nachfrage-Verlagerung für das Wachstum in Europa und Asien habe, hänge hauptsächlich von den Entscheidungen in der Finanz- und Währungspolitik in den betroffenen Ländern ab, sagte Dullien. Er erwarte nicht, dass die Europäische Zentralbank deutlich auf den Nachfrage-Rückgang aus Amerika reagieren würde. Dullien kritisierte in diesem Zusammenhang scharf die Geldpolitik der EZB, vor allem ihre Orientierung an der Geldmenge M3. Auch sei von Seiten der Finanzpolitik nicht viel zu erwarten, weil der Stabilitätsund Wachstumspakt der Europäischen Union die Handelsspielräume der Finanzminister zu stark einschränke. Er schlage deshalb vor, den Pakt im Falle einer starken Aufwertung des Euro ganz außer Kraft zu setzen. In Asien dagegen hänge fast alles von der Währungspolitik der einzelnen Länder ab und davon, ob es Volkswirtschaften wie China gelingen könne, ihre Binnennachfrage anzukurbeln.

Adam Posen stimmte mit fast allen von Dulliens Aussagen überein. Er sagte jedoch, dass die Folgen einer Berichtigung des amerikanischen Leistungsbilanzdefizits nicht so sehr von der Wirtschaftspolitik einzelner Länder abhingen, sondern von den institutionellen Wirtschaftsstrukturen in Europa und Asien. Wie Dullien konnte Posen der Geldpolitik der Europäischen Zentralbank nichts Gutes abgewinnen. Er betonte, dass ein übergreifender 
europäischer automatischer Stabilisator, wie zum Beispiel eine europaweite Arbeitslosenversicherung, die Anpassung des Euroraumes an einen amerikanischen Nachfragabfall vereinfachen könnte. Auch er gehe davon aus, dass eine solche Anpassung wesentlich schmerzhafter für Europa sei als für andere Teile der Weltwirtschaft.

Carl-Ludwig Holtfrerich zeigte sich in seinem Kommentar zu Dulliens und Posens Beiträgen überrascht, dass so viele Ökonomen sich ausschließlich auf das Leistungsbilanzdefizit der Amerikaner konzentrierten. Die ausgeglichene Leistungsbilanz der Europäischen Union gebe ihm viel mehr zu denken. Die Aussicht, dass diese Bilanz in der nahen $\mathrm{Zu}$ kunft in einen wesentlichen Leistungsüberschuss umschlagen könne, gebe noch mehr Anlass zu Sorge. So seien doch Defizitländer hauptsächlich von starkem Wirtschaftswachstum, Investitionsschüben und hohem Produktivitätswachstum gekennzeichnet, während Überschussländer mit schleppenden Wachstumsraten und einem Rückgang in Pro-Kopf Einkommen und sinkenden Lebensstandards zu kämpfen hätten. Im Übrigen hielte er die Sorge vieler Wirtschaftswissenschaftler angesichts der weltwirtschaftlichen Ungleichgewichte für übertrieben: Das Ende der BrettonWoods Ära und die Öffnung nationaler Kapitalmärkte habe es doch gerade ermöglicht, dass dynamischere Volkswirtschaften auf die Ersparnisse stagnierender Länder zurückgreifen könnten - zum Vorteil aller in der Weltwirtschaft.

Andere Teilnehmer konterten, dass die Sorge vieler Ökonomen um das amerikanische Leistungsbilanzdefizit vor allem mit der wachsenden Außenverschuldung des Landes zusammenhänge. Und auch wenn viele Wissenschaftler eine ,milde“ Anpassung der Weltwirtschaft an eine Abwertung des Dollarkurses erwarteten, könne diese Anpassung immerhin noch ein Prozent der gesamten Weltwirtschaftsleistung kosten - mit schmerzhaften Folgen für viele Länder.
Vom Weltbanker, dem amerikanischen Immobilienboom und der produktivsten Volkswirtschaft der Welt

Catherine Manns und Katharina Plücks Beitrag befasste sich vor allem mit der detaillierten Struktur der derzeitigen amerikanischen Außenverschuldung, und inwieweit diese Auskunft über den möglichen Anpassungsprozess der amerikanischen Wirtschaft an Wechselkurs- und Zinssatzveränderungen gibt. Da die amerikanische Wirtschaft die Welt mit einer internationalen Währung versorge habe Amerika bisher den Vorteil gehabt, Schulden in Dollar aufzunehmen und Kredite in anderen Währungen zu vergeben. Senkungen des Dollarkurses hätten so bisher die Außenverschuldung der USA in großem Maße stabilisiert. Außerdem sei bekannt, dass es den USA noch immer gelinge, trotz einer Netto-Verschuldung von 22 Prozent des Bruttoinlandproduktes weiterhin positive NettoEinkommenströme zu verzeichnen. Das hinge zum einen damit zusammen, dass die USA wie eine Bank oder ein großer Risikokapitalanleger kurzzeitige Kredite zu niedrigen Zinssätzen aufnehmen und diese dann in langfristige, risikoreiche Auslandsinvestitionen mit hohen Renditen anlegen. Zudem schienen die amerikanischen Direktinvestitionen im Ausland, Datenzeitreihen zufolge, deutlich rentabler zu sein als die ausländischen Direktinvestitionen in den USA. All diese Faktoren sprächen also für eine langfristige Nachhaltigkeit einer wachsenden amerikanischen $\mathrm{Au}-$ Benverschuldung und gäben relativ wenig Grund zur Sorge.

Jedoch zeigten Mann und Plück, dass vor allem neuere Daten eine Trendwende in der amerikanischen Außenverschuldung anzeigten: Die Renditedifferenzen zwischen ausländischen und amerikanischen Direktinvestitionen seien in letzter Zeit geschrumpft. Amerikas Abhängigkeit von internationalen Investitionen in festverzinsliche Schuldbriefe berge in Zeiten von Zinssatzerhöhungen einige Risiken. Man müsse sich auch fragen, wann ausländische Investoren, besorgt um be- 
vorstehende Dollarkursverluste, wesentliche Risikoprämien verlangten - mit negativen Konsequenzen für die Investitionsquote und das Wirtschaftswachstum der USA. Auch sei es vorstellbar, dass der Wertpapierbestand ausländischer Anleger bei wachsender amerikanischer Außenverschuldung irgendwann zu viele auf Dollar lautende Komponenten enthalte und Investoren ihre Portfolios zu Gunsten anderer Währungen umschichteten. Das hätte dann weitere negative Konsequenzen für Risikoprämien und den Dollarkurs.

Vor allem in letzter Zeit hieß es oft, dass die amerikanische Außenverschuldung vor allem die anhaltende Importnachfrage der USA nach Konsumgütern und eine zunehmend ineffiziente Immobilienblase finanziert hat. In seinem Vortrag über den amerikanischen Hypothekenmarkt machte Martin Mühleisen jedoch deutlich, dass in diesem Zusammenhang momentan noch wenig Grund zu Sorge bestehe. Amerikanische Finanzmärkte seien äuBerst dynamisch und flexibel und immer auf der Suche nach neuen Produkten für Anleger. Deregulierung habe die Risiken von Hypotheken weitestgehend diversifiziert und Immobilienmärkte seien durch „De-Regionalisierung" weitaus berechenbarer als früher. Das spiegele beispielsweise die Immobilienwirtschaft wieder, die in letzter Zeit ihre Vorratsbestände weniger stark aufbaue.

Für ausländische Investoren böten amerikanische Hypotheken sichere und sehr rentable Anlagen. Natürlich könnte es immer noch passieren, dass eine Anhebung der Zinssätze einen Einbruch im amerikanischen Immobiliengeschäft herbeiführen könne. Dies sei in der Vergangenheit allerdings vor allem in Zeiten hoher Arbeitslosigkeit passiert und deshalb in naher Zukunft weniger wahrscheinlich. Die Verschuldung von amerikanischen Privathaushalten erscheine weiterhin stabil und überschaubar - vor allem weil die Gewährung riskanter Immobilienkredite stark zurückgegangen sei. Mühleisen glaubte deshalb nicht, dass ein Zinsanstieg zwangsläufig zu einer amerikanischen Rezession führe. Auch halte er, wie er nachdrücklich betonte, die Anlagen ausländischer Investoren in den amerikanischen Immobilienmarkt nicht für ineffizient.

Kent Hughes hingegen zeigte sich besorgt darüber, was genau die amerikanische Außenverschuldung in den letzten Jahren finanziert habe. Zwar sei die amerikanische Volkswirtschaft bisher die dynamischste und produktivste in der Welt. Aber diese Vorrangstellung sei nicht auf ungewisse Zeit garantiert. Das ansteigende amerikanische Haushaltsdefizit stelle eine konstante Bedrohung für den technologischen Fortschritt im Lande dar. Denn trotz dieses steigenden Defizits seien in den letzten zehn Jahren viel zu wenig Gelder in das öffentliche Bildungssystem, die Grundlagenforschung und den Schutz gewerblicher Urheberrechte, vor allem China gegenüber, geflossen. Zudem sehe sich Amerika mit einem wachsenden weltweiten Wettbewerb um Talente konfrontiert: China und Indien gewinnen mehr und mehr Mitglieder ihrer gut ausgebildeten Diaspora in den USA zurück und bilden mittlerweile selbst hochqualifizierte Wirtschaftskräfte aus. Wenn Amerika weiterhin im weltweiten Wettbewerb mithalten und gar eine wirtschaftliche Führungsrolle spielen wolle, müsse viel mehr Geld in die Wettbewerbsfähigkeit amerikanischer Arbeitskräfte gesteckt werden. Amerikanische Politiker müssten allerdings auch in Foren wie der Welthandelsorganisation auf gerechten weltweiten Wettbewerb pochen. Um wirtschaftliche Ungleichgewichte zu beseitigen, kann Hughes sich gut ein zweites Plaza Abkommen vorstellen, das eine konzertierte Korrektur des amerikanischen Leistungsbilanzdefizits und des Dollar-Wechselkurses erlaube.

In ihrem Kommentar zu Hughes', Mühleisens, Manns und Plücks Vorträgen erklärte Stormy Mildner, dass der Davos ,Global Competitiveness Index ' die Schwächen in der amerikanischen Wirtschaft in der makroökonomischen Säule, vor allem dem wachsenden Leistungsbilanzdefizit der USA, ansiedele. Sie glaube nicht, dass der internationale Wäh- 
rungsfond zur Auflösung dieser wirtschaftlichen Ungleichgewichte beitragen könne. Mildner hob auch die Verflechtungen der ersten makroökonomischen Säule des Indexes und der mikroökonomischen technologischen Vorrangstellung der amerikanischen Wirtschaft hervor: So trügen die hervorragende Dynamik der US Wirtschaft und ihre Attraktivität für ausländische Investoren ja die Verantwortung für anhaltende Kapitalzuflüsse und verschlimmerten so Ungleichgewichte in der Weltwirtschaft.

Was sollte Europa tun? Besser regieren und kein Geld verschwenden

Dass die europäische Wirtschaft von einer solchen Dynamik nur träumen kann, darin waren sich alle Konferenzteilnehmer einig. Stefan Collignon führte diesen Mangel an Dynamik vor allem auf eine völlig falsche Geldund Finanzpolitik im Euroraum zurück. In seinem Vortrag machte er deutlich, dass, weil es innerhalb der Währungsunion keine gemeinsam festgelegte Finanzpolitik gebe, die Kombination von überregionaler Geld- und auseinanderweichender nationaler Wirtschaftspolitik verheerende Konsequenzen für die Zinsentwicklungen im Euroraum gehabt habe. Ohne eine übergreifende europaweite Regierung sehe er keine Chance, dass Europa in naher Zukunft an das Produktivitätswachstum der amerikanischen Wirtschaft heranreichen könne. Ohne Regierung, die nicht nur für die Wirtschafts- und Geldpolitik der gesamten Währungsunion, sondern auch für die Formulierung eines europäischen außenpolitischen Interesses verantwortlich sei, könne Europa nie die nötigen Ressourcen und die politische Willenskraft aufbringen, die Verantwortung für globale Sicherheit mit den Vereinigten Staaten zu teilen.

Daniela Schwarzer teilte Collignons Auffassung. Es seien vor allem anhaltende nationale Interessen, die die Entwicklung einer wirklich effektiven gemeinsamen europäischen Verteidigungspolitik zurückhielten. Schwarzer zeigte, dass europäische Militäraufwendun- gen stagniert haben oder gar zurückgegangen sind, während amerikanische Militärausgaben seit dem 11. September 2001 stark angestiegen sind. Die unterschiedlichen Auffassungen in Amerika und Europa zur Bedeutung militärischer Macht für die globale Sicherheit lieBen sich auch an den verschiedenen Sicherheitsstrategien der Partner ablesen. Trotzdem, sagte Schwarzer, könnten die Europäer ihre militärische Beteiligung an internationalen Sicherheitsbemühungen stark erhöhen und verbessern: Dazu müssten sie ineffiziente Doppelungen bei Rüstungsbeschaffungen vermeiden, vorhandene Militärausgaben effektiver einsetzten und nationalistischen Tendenzen vor allem in Bezug auf die europäische Rüstungsindustrie entgegenwirken. Wie Collignon befürwortete Schwarzer eine übergreifende europäische Regierungsinstanz, die den Willen der europäischen Wähler in Sachen integrierter Verteidigungs- und Außenpolitik legitimer ausführen könnte.

Tilman Brück kritisierte in seinem Kommentar Collignons Vorschlag einer gemeinsamen europäischen Wirtschaftspolitik. Gesunde Wirtschaftpolitik könne auch auf der Ebene der europäischen Nationalstaaten für ein insgesamt stärkeres Wirtschaftswachstum sorgen. Allerdings teilte er Collignons Auffassung, dass der Stabilitäts- und Wachstumspakt der wirtschaftlichen Entwicklung im Euroraum bisher eher hinderlich gewesen sei. Wie Collignon und Schwarzer vor ihm unterstrich Brück die Tatsache, dass Europa und die USA die Probleme internationaler Sicherheit grundsätzlich verschieden angingen. Auch er sehe in nächster Zukunft keine Annäherung zwischen den verschiedenen Vorgehensweisen der transatlantischen Partner.

Transatlantische Beziehungen: Immer noch eine ,Special Relationship'?

Brücks pessimistischer Ausblick hinsichtlich dieser verschiedenen Vorgehensweisen wurde von Stephen Szabos und Reinhard Wolfs Beiträgen bekräftigt. Dass Europa sich weniger 
militärisch engagiert zeige, sei allein durch die schmerzhafte neueste Geschichte des Kontinents zu erklären, sagte Szabo. Allerdings erschiene es so, als seien die Werte auf beiden Seiten des Atlantiks nach dem 11. September noch weiter auseinandergerückt. Die Europäer hätten in den letzten Jahren ihr Vertrauen in die amerikanische Außenpolitik verloren, und die Amerikaner sähen sich zunehmend isoliert in der Welt.

Dieser Vertrauensverlust zeigte sich deutlich in den deutschen und europäischen Meinungsumfragen, die Wolf in seinem Vortrag präsentierte. Europäische Befragte zeigten sich weit weniger besorgt über internationalen Terrorismus oder den Krieg im Irak als amerikanische Bürger. Wirtschaftliche und soziale Sorgen nehmen im Wahlverhalten der Europäer einen viel größeren Stellenwert ein. So sei natürlich die Unterstützung für größere Militärausgaben in Europa nicht groß. Mehr noch: Europäer kritisierten den hohen Militäraufwand der Vereinigten Staaten. Die Befragten äußerten, dass die Amerikaner mit ihrem Vorgehen die Welt unsicherer gemacht hätten.

Während viele amerikanische Konferenzteilnehmer die zaghafte europäische Verteidigungspolitik scharf kritisiert und die Europäer als ,free rider' kritisiert hatten, hob Szabo hervor, dass die USA trotz militärischer Vormacht auf Kooperation mit den europäischen Alliierten angewiesen seien. Europäische Unterstützung amerikanischer Außenpolitik bedeute eine Legitimierung der USA gegenüber dem Rest der Welt. Es sei fraglich, ob die USA ohne europäische Verbündete wirklich eine Weltmacht darstellen könnten. Wolf sagte, dass sich die USA vor dem Hintergrund europäischen Misstrauens wieder mehr auf Konsultation mit den Europäern einlassen müssten. Im Gegenzug könnten die Europäer von öffentlicher Kritik der amerikanischen Außenpolitik absehen und - wenn auch ihre Verteidigungsbudgets in den nächsten Jahren nicht wüchsen - amerikanischen globalen Politikzielen mehr zivile Mittel zur Verfügung stellen.

Szabo bemängelte, dass eine gemeinsame europäische Außenpolitik zu wenig institutionalisiert sei. Dies mache erfolgreiche transatlantische Zusammenarbeit zu sehr von den Führungspersönlichkeiten auf beiden Seiten des Ozeans abhängig. Zurzeit seien zu viele europäische Staatsoberhäupter innenpolitisch lahm gelegt, und Präsident Bush werde in Europa als starke Führungsperson nicht länger wahrgenommen. Szabo erhoffte sich deshalb, dass die Nato in Zukunft wieder eine effektive und ernstzunehmende transatlantische Institution bilden könne.

In seiner Zusammenfassung der Konferenz bekräftigte auch Nau sein grundsätzliches Vertrauen in das Fortbestehen der transatlantischen Allianz. Die Konferenz habe deutlich gemacht, dass europäische und amerikanische Wissenschaftler sehr unterschiedliche Meinungen vertreten, wie die Lasten in den Bemühungen um globale Sicherheit besser und gerechter verteilt werden könnten beziehungsweise ob ein Lastenausgleich überhaupt notwendig sei. Trotzdem bestehe weiterhin ein starker Glaube an die Fähigkeit der Allianz, Veränderungen im Weltgeschehen und zukünftige Herausforderungen an die Weltsicherheit intakt zu bestehen. Das transatlantische Bündnis, so Nau, sei und bleibe etwas Besonderes. Der „demokratische Friede“ sei ein Phänomen, das Politikwissenschaftler immer noch nicht zu erklären wüssten. Aber es gebe ihn, und er sei evident in den außenpolitischen Beziehungen zwischen Europa und den Vereinigten Staaten von Amerika. 